\title{
Transcranial direct current stimulation to prevent and treat surgery-induced opioid dependence: a systematic review
}

\section{Pain Management}

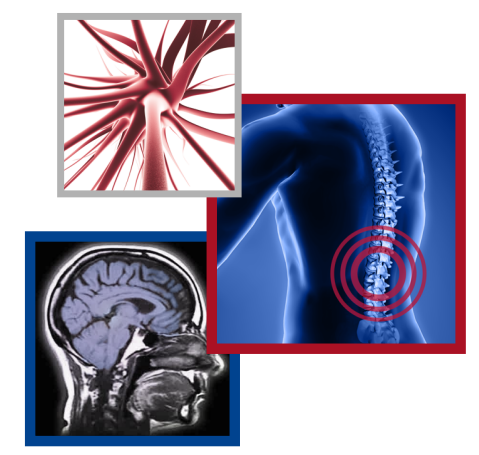

\author{
Alessia Gallucci*,1,2, Pedro H Lucena ${ }^{1,3}$, Géraldine Martens ${ }^{4}$, Aurore Thibaut ${ }^{1,4}$ \& Felipe \\ Fregni ${ }^{1}$ \\ ${ }^{1}$ Neuromodulation Center, Spaulding Rehabilitation Hospital, Harvard Medical School, Boston, MA, 02129, USA \\ ${ }^{2}$ Department of Psychology, University of Milano-Bicocca, Milan, Italy \\ ${ }^{3}$ Department of Medice, Bahiana School of Medicine \& Public Health, Salvador, BA, Brazil \\ ${ }^{4}$ Coma Science Group, GIGA Research \& Neurology Department, University \& University Hospital of Liege, Liege, Belgium \\ *Author for correspondence: fregni.felipe@mgh.harvard.edu
}

Opioid misuse leading to dependence is a major health issue. Recent studies explored valid alternatives to treat pain in postsurgical settings. This systematic review aims to discuss the role of transcranial direct current stimulation (tDCS) in preventing and treating postoperative pain and opioid dependence. PubMed and Embase databases were screened, considering studies testing tDCS effects on pain and opioid consumption in surgical settings and opioid addiction. Eight studies met our inclusion criteria. Results showed a reduction of postoperative pain, opioid consumption and cue-induced craving following cortical stimulation. Despite the limited number of studies, this review shows preliminary encouraging evidence regarding the analgesic role of tDCS. However, future studies are needed to further investigate the application of tDCS in postsurgical settings.

First draft submitted: 17 July 2018; Accepted for publication: 24 September 2018; Published online: 5 December 2018

Keywords: opioid dependence $\bullet$ postoperative pain $\bullet$ preventing $\bullet$ tDCS • treating

The opioid crisis is arguably one of the most serious public health problems of our time. One of their main prescription uses is in the postsurgical setting. However, misuse leading to opioid dependence, and eventually to overdose death, has raised concerns regarding the impact of opioid use on public health. In 2015, over 33,000 deaths from opioid overdose were reported in the USA (63.1\% of all drug overdose deaths) [1]. Epidemiological studies have shown that this rate has increased since the year 2000 [2]. Alarmingly, this rise of deaths from overdose is paralleled by the increasing rate of opioid prescriptions. Patients undergoing surgery are four-times more likely to be discharged with an opioid prescription than other patients. This major public health issue seems to be confined to the USA, which is by far the world leader in opioid consumption [3]. There is evidence of a strong relationship between the amount and duration of prescriptions and subsequent opioid misuse in the surgical population. About $1 \%$ of patients receiving opioids in a postsurgical setting will indeed develop dependence. Interestingly, the duration of prescription has more of an impact on the risk to develop dependence than the doses themselves [4]. Opioids being overprescribed by physicians appear as a serious contributing factor to the overdose mortality rate [5]. Alternatives to manage pain appropriately therefore are indeed crucial to tackle this major crisis and to improve patients' care.

The most common strategy to face opioid dependence is a pharmacological intervention (for a review, see [6]). Mainly, the methadone maintenance treatment, consisting of daily administration of the oral opioid agonist methadone, is a well-established and cost-effective treatment [7-9]. Recently some alternatives have been effectively tested, such as longer-acting agonist lambda- $\alpha$-acetylmethadol and the mixed agonist/antagonist buprenorphine [10]. However, the effectiveness variability, restricted admissions in treatment programs, high rates of relapse, presence of several side effects as well as the high risk of mortality linked to methadone overdose interfere with the dependence recovery [11-15]. In addition, the mechanisms of these drugs may be diffuse. In fact, dependence mechanisms involve different dopaminergic structures, which are all part of the reward system: the prefrontal cortex, the ventral tegmental area and its projections to the nucleus accumbens [16]. While pharmacological treatments are showing

Future $\because$ Medicine 
their limits, new therapeutic options that are more targeted to the dysfunctional neural circuits are being explored, such as noninvasive brain stimulation $[17,18]$.

Transcranial direct current stimulation (tDCS) is a technique of noninvasive brain stimulation that has already shown its efficiency in drug-avoiding behavior $[19,20]$. TDCS can be used to inhibit craving behavior or to control pain, depending on the area of stimulation, thus treating and preventing opioid dependence, respectively. Initial neurophysiological findings have shown bidirectional, time- and polarity-dependent excitability changes following the administration of weak electrical currents to the brain [21,22]. TDCS transiently modifies spontaneous cortical excitability by delivering low-intensity electrical currents to the scalp. Anodal stimulation depolarizes neurons' resting membrane potentials, enhancing neural excitability, while cathodal stimulation causes the opposite effect $[21,23,24]$. In addition to those effects that occur during and immediately after the stimulation, tDCS also results in prolonged effects that involve NMDA receptors as well as long-term potentiation and long-term depression mechanisms [21,25]. Thus, tDCS can be used to induce long-lasting modulation on neural areas associated with addiction and pain.

Regarding addiction, several studies demonstrated that single or repetitive tDCS sessions applied over the prefrontal cortex significantly reduced cue-provoked smoking and crack-cocaine craving [19,26-29] as well as alcohol craving [30]. Prefrontal stimulation can also affect the activity of the limbic system, modulating the emotional components of pain $[31,32]$. For pain management, the primary motor cortex is a popular target for tDCS stimulation [31,33-37]; it is hypothesized to change the activity of the thalamus [32,38,39] and pain pathway areas [23] as well as inhibit the somatosensory cortex [40-42]. Moreover, several studies demonstrated the impact of serotonin as a pain relief agent $[43,44]$ and the role of the opioid system in the analgesic mechanism of $\mathrm{tDCS}[45,46]$.

In this review, we discuss the potential of tDCS to combat the opioid epidemic by reviewing its use to: first, prevent opioid addiction by using tDCS as a presurgical procedure; or in the immediate postsurgical period, and second, treat opioid addiction while using opioids.

\section{Methods}

We conducted this systematic review following the Preferred Reporting Items for Systematic Reviews and MetaAnalyses Protocols [47,48].

\section{Search strategy \& eligibility criteria}

We performed two different searches to discuss tDCS as a tool to prevent and treat postoperative pain and opioid dependence. The strategies shown in Appendix 1 were used to collect records from PubMed and Embase. We considered the following eligibility criteria: clinical trials, defined by the NIH as "a research study in which one or more human subjects are prospectively assigned to one or more interventions (which may include placebo or other control) to evaluate the effects of those interventions on health-related biomedical or behavioral outcomes" [49]; studies performed in humans; studies with tDCS and surgery or tDCS and opioid dependence; single or multiple tDCS sessions; studies with validated outcome measures; papers published in English; papers published after 2000; and peer-reviewed papers.

\section{Screening \& selection of records}

After identifying and excluding duplicates, two researchers performed independent filters based on title and abstract. When title/abstract lead to exclusion of the paper, the investigator used labels to justify the reason of exclusion. To ensure the blinding of the process, we used Rayyan, a web and mobile systematic reviews manager [50]. At the end of this process, the blind mode was turned off and conflicts were solved by a third evaluator. The included papers were reviewed and screened based on the inclusion and exclusion criteria.

\section{Data extraction}

Data were extracted using two structured checklists, one for 'tDCS to prevent and treat postoperative pain' and the other for ' $\mathrm{tDCS}$ to treat opioid dependence'. Both of them included the following variables: bibliographic details of papers (author, title of study, journal, year and country); trial characteristics (design, duration, number of followups, eligibility criteria, limitations and adverse events reported); subjects' demographics (number of participants, age and gender); tDCS parameters (device, montage, number of sessions and frequency, stimulation duration, current intensity, electrodes size); the primary and secondary outcomes measures; and results. For the 'tDCS to prevent and treat postoperative pain' search, we also considered the type of surgery and opioid used during the 


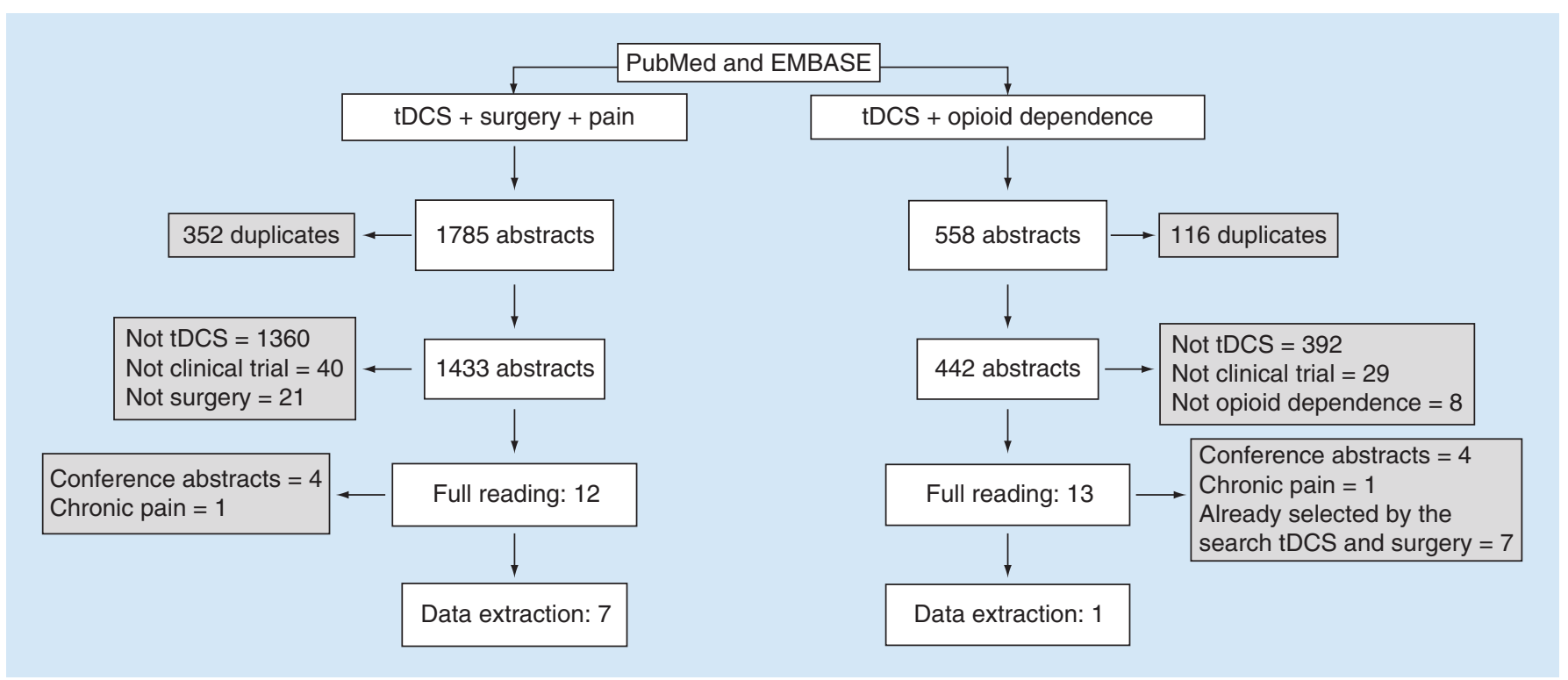

Figure 1. Flowchart showing the selection process of papers. The right side of the flowchart represents the selection process related to the search 'transcranial direct current stimulation to prevent and treat postoperative pain' while the left represents the search process of 'transcranial direct current stimulation to treat opioid dependence'. Gray boxes indicate papers excluded during the filtering process.

postoperative period. For the 'tDCS to treat opioid dependence' search, we added the kind of opioid prescribed and the mean of dependence time.

\section{Results}

The search 'tDCS to prevent and treat postoperative pain' retrieved 1785 papers ( 455 from PubMed and 1330 from Embase). After excluding the duplicates, selecting the papers considering title and abstract, and solving the conflicts, 12 papers were included to the full reading. Among them, five studies were excluded (four conference abstracts and one related to chronic pain) and seven papers were included to the data extraction. Regarding the search 'tDCS to treat opioid dependence', 558 abstracts were collected (153 from PubMed and 405 from Embase). After the first filter process, 13 papers were selected for the full reading. Among them, we excluded 12 papers (four conference abstracts, one related to chronic pain and seven that had already been selected by the search tDCS and surgery pain). The results of the search strategy are summarized in Figure 1.

\section{Results 'tDCS to prevent \& treat postoperative pain'}

We evaluated seven clinical, randomized and sham-controlled trials [51-57]. Two of them used a single-blinded design [53,54] and five were double-blinded [51,52,55-57]. Four studies were done in the USA [53-56] while the other three were carried out in Brazil [57], Egypt [52] and Belgium [51]. The papers were published between 2011 and 2017.

The studies included 292 subjects in total. The mean age ranged from 37 to 67 years and 195 subjects $(66.8 \%)$ were female. A total of 147 subjects were submitted to total knee arthroplasty, 86 to lumbar spine surgery, 40 to hallux valgus surgery and 19 to endoscopic retrograde cholangiopancreatography. Thus, the types of surgeries varied significantly across studies.

The strategy of stimulation also varied significantly across these studies; we can divide them into two main categories: stimulation of the motor cortex (four studies); and stimulation of the prefrontal cortex (two studies). One study tested both strategies in a four-group design (motor cortex vs dorsolateral prefrontal cortex vs control site vs sham) [55]. One important point to mention is that the studies utilizing primary motor cortex (M1) stimulation differed in respect to the return electrode: contralateral supra-orbital area (one study), contralateral dorsolateral prefrontal cortex (DLPFC) (two studies) and extracephalic (one study). Most of the studies used anodal polarity over the M1. For the DLPFC strategy, one study [54] placed the reference electrode at contralateral somatosensory cortex, whereas another study [51] placed the reference electrode on the scalp over the right ear in both conditions (Table 1). 


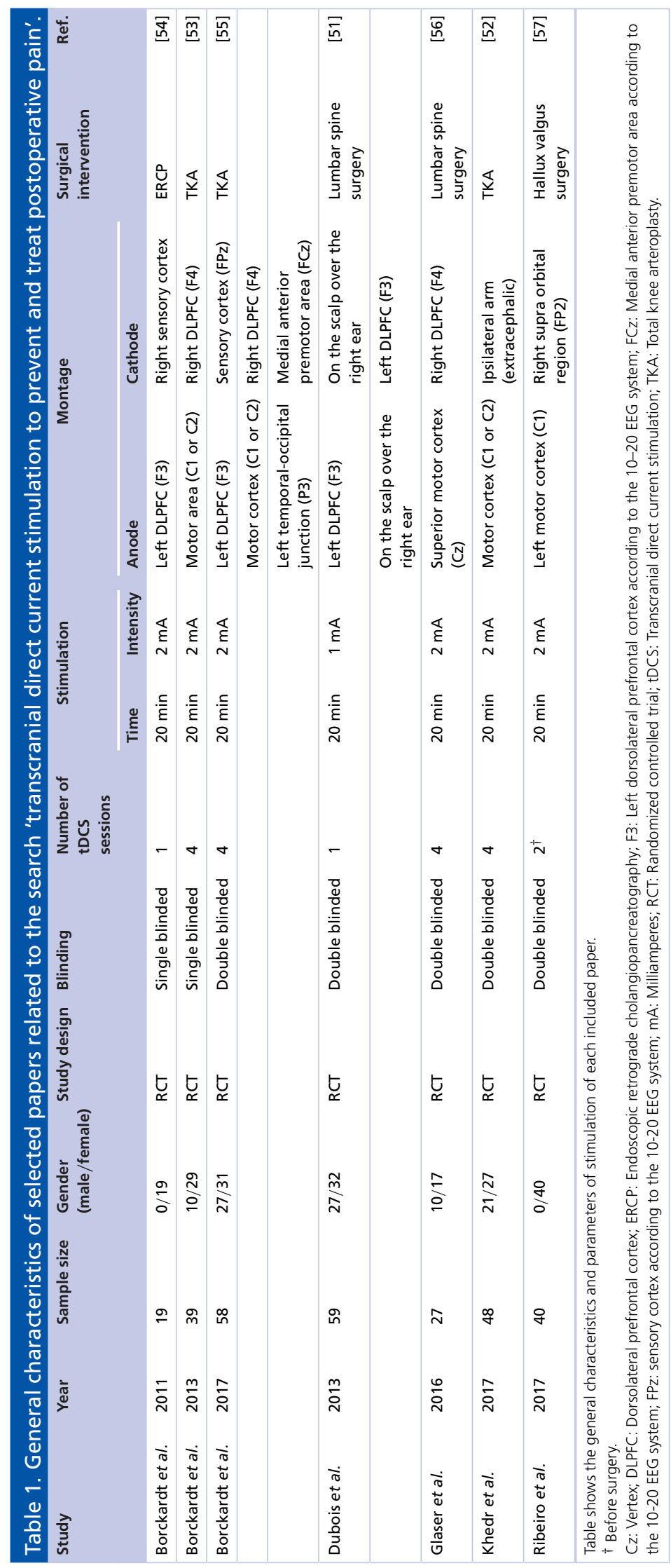


Table 2. Results of selected papers related to the search 'transcranial direct current stimulation to prevent and treat postoperative pain'.

\begin{tabular}{|c|c|c|c|c|c|c|}
\hline \multirow[t]{2}{*}{ Study } & \multirow[t]{2}{*}{ Year } & \multirow[t]{2}{*}{ Sample size } & \multicolumn{3}{|c|}{ Pain } & \multirow[t]{2}{*}{ Ref } \\
\hline & & & VAS at rest & VAS dynamic & PCA & \\
\hline Borckardt et al. & 2011 & 19 & $\begin{array}{l}\text { No differences (arithmetic } \\
\text { advantages toward stimulation } \\
\text { group) }\end{array}$ & - & $22 \%$ less $(p=0.0003)$ & {$[54]$} \\
\hline Borckardt et al. & 2013 & 39 & No differences & - & $46 \%$ less $(p=0.006) . E S=0.95$ & [53] \\
\hline \multirow[t]{3}{*}{ Borckardt et al. } & 2017 & 58 & No differences & - & $\begin{array}{l}\text { Anode over F3 vs sham: } 24 \% \text { less } \\
(p<0.0001) . E S=0.365\end{array}$ & [55] \\
\hline & & & & - & $\begin{array}{l}\text { Anode over } M 1 \text { vs sham: } 27 \% \\
\text { more }(p<0.0001) . E S=-0.323\end{array}$ & \\
\hline & & & & - & $\begin{array}{l}\text { Anode over P3 vs sham: no } \\
\text { differences }\end{array}$ & \\
\hline \multirow[t]{3}{*}{ Dubois et al. } & 2013 & 59 & $\begin{array}{l}\text { F3 cathodal vs sham: } 43 \% \text { less } \\
(p=0.150)\end{array}$ & $\begin{array}{l}\text { F3 cathodal vs sham: } 24.6 \% \text { less } \\
(p=0.245)\end{array}$ & $\begin{array}{l}\text { F3 cathodal vs sham: } 32 \% \text { less } \\
(p=0.258) . E S=0.53\end{array}$ & [51] \\
\hline & & & $\begin{array}{l}\text { F3 anodal vs sham: } 42 \% \text { less } \\
(p=0.093)\end{array}$ & $\begin{array}{l}\text { F3 anodal vs sham: } 10.4 \% \text { less } \\
(p=0.675)\end{array}$ & $\begin{array}{l}\text { F3 anodal vs sham: } 7.6 \% \text { more } \\
(p=0.771) . E S=-0.085\end{array}$ & \\
\hline & & & $\begin{array}{l}\text { F3 cathodal vs anodal: } 1.7 \% \text { less } \\
(p=0.879)\end{array}$ & $\begin{array}{l}\text { F3 cathodal vs anodal: } 15.9 \% \text { less } \\
(p=0.421)\end{array}$ & $\begin{array}{l}\text { F3 cathodal vs anodal: } 36.6 \% \text { less } \\
(p=0.445) . E S=0.5\end{array}$ & \\
\hline Glaser et al. & 2016 & 27 & - & - & $23.6 \%$ less $(p<0.001) . E S=0.3$ & [56] \\
\hline Khedr et al. & 2017 & 48 & $5 \%$ less $(p=0.411)$ & - & $26 \%$ less $(p=0.0002) . E S=0.646$ & [52] \\
\hline Ribeiro et al. & 2017 & 40 & Less pain $(p<0.001)$ & Less pain $(p<0.001)$ & $\begin{array}{l}73.25 \% \text { less }(p<0.001) \\
E S=0.692\end{array}$ & [57] \\
\hline
\end{tabular}

Regarding the number of tDCS sessions, a single one was applied in two studies [51,54] and two or more tDCS sessions in five other studies [52,53,55-57]. Six studies [51-56] performed tDCS immediately after surgery and only one study [57] applied tDCS prior to the surgery. The duration of stimulation was the same; however, current intensity differed among studies. Six out of seven studies [52-57] applied $2 \mathrm{~mA}$ stimulation and one [51] applied $1 \mathrm{~mA}$ stimulation.

Five out of seven papers [51,52,54-56] reported adverse events and none of them were considered serious (Appendix 3). Tingling, itching, mild stinging and burning sensations were the most common adverse events. Dubois et al. [51] also described a visual flash sensation experienced by one of the subjects (out of 20 in the cathodal stimulation group) at the start of the stimulation.

In terms of the results from these clinical trials, the most clear and significant results came from patient-controlled analgesia (PCA). This outcome measures the usage of self-administered opioids. In five studies [52-54,56,57], a reduction of the PCA was observed varying from 22 to $73.25 \%$, and the differences were statistically significant. In Dubois et al. [51], however, no statistically significant differences were observed in relation to this parameter. And in Borckardt et al. [55], the group in which the anode was positioned over F3 presented a reduction of $24 \%$ in the consumption of opioids, while the group with the anode positioned over motor cortex (C1 or C2) showed an increase in consumption by $27 \%$ as compared with the sham, both differences with $\mathrm{p}<0.05$. Given the limited data, it is difficult to state that M1 stimulation in this study resulted in increased opioid use compared with other studies. Authors speculated that the observed difference in the first scenario is due to the fact that the cathodal stimulation of the right DLPFC might have limited the pain relief role of the M1 anodal stimulation; in the second scenario, authors hypothesized that the cathodal stimulation of the sensory cortex might have enhanced the analgesic effects of the left DLPFC anodal stimulation. Finally, visual analog scale results were only significant in one study [57] that measured the tDCS analgesic effects considering pain scores at rest and during walking for the first 48 postoperative hours and throughout the 7-day postoperative period. Mainly, active tDCS, compared with sham stimulation, resulted in less pain in both conditions in all time points considered (Table 2).

\section{Results 'tDCS to treat opioid dependence'}

The search for studies assessing the impact of tDCS on opioid dependence and misuse retrieved only one new paper [58]. The randomized, single-blinded and sham-controlled study was conducted in China and included 
individuals who experienced addiction for $>3$ continuous years and were abstinent from heroine for at least 1.5-2 years. Twenty male subjects with a mean age of 39.8 (standard deviation $[S D]=1.8$ ) and history of $5-25$ years of heroin dependence (mean $=16.9$ years, $\mathrm{SD}=1.1$ ) were randomly assigned to receive active (cathode over T3 and $\mathrm{T} 4$, and anode over $\mathrm{O} 2$ and $\mathrm{O} 1$ ) or sham stimulation. A single stimulation session lasting 20 min with a current intensity of $1.5 \mathrm{~mA}$ was applied. As the primary outcome, investigators exposed the subjects to a real video of heroin use and asked them to fill out the craving questionnaire before and after $\mathrm{tDCS}$. The group that received active stimulation showed a decrease of $36.7 \%$ on the craving score $(\mathrm{p}=0.003$ ), while no difference between pre and post $\mathrm{tDCS}$ stimulation was observed in the sham group.

\section{Discussion}

This review aimed to identify the level of evidence about the effect of tDCS in preventing and treating postsurgical pain and opioid dependence. We performed two different searches and eight papers were retrieved.

The studies selected appear quite different in regards to the stimulation procedure, suggesting that there are different strategies for the treatment of pain and prevention of opioid dependence using noninvasive stimulation. We observed that all studies included in this review performed $20 \mathrm{~min}$ of tDCS stimulation at a current intensity of 1-2 mA (in line with the safety standards) [59], and positioned electrodes following the 10-20 EEG system or its variations. However, the current density, the montage and the number of intervals between sessions were not standardized across studies.

All eight studies included in the present review targeted mainly two brain areas, the M1 and the DLPFC. As aforementioned, these two regions play a crucial role in pain and opioid addiction. While M1 is believed to modulate the somatosensory system and thus reduce overactivation in these circuits, the DLPFC (also part of the inhibitory control system) is believed to modulate the affective aspects of pain [60-63]. The DLPFC is also important for both pain control $[62,64,65]$ and reward responses modulation, representing a crucial target when dealing with craving [66]. The literature has supported the role of mesolimbic and mesocortical dopamine pathways in reward circuit, especially the tegmental area and the nucleus accumbens $[67,68]$ as well as the amygdala and the hippocampus, involved in generating conditioned responses and underlying emotional memories and the orbitofrontal cortex, crucial for computing the value of stimuli and driving motivation $[68,69]$. Overall, the studies analyzed here support the idea that tDCS strengthens top-down regulation processes in both pain modulation and opioid dependence [55,56,58].

Regarding pain modulation, stimulation of the DLPFC could affect the limbic structures, resulting in a better control of emotional components of pain $[31,32,70,71]$. TDCS over M1 could have indirect effects on the thalamus, modulating pain-related areas and nociceptive pathways [34,72,73]. This pain control might in turn contribute to the reduction of opioid consumption. The potential postoperative pain reduction role of the neuro stimulation techniques has been also investigated with repetitive transcranial magnetic stimulation. In two studies, repetitive transcranial magnetic stimulation was applied over the left DLPFC in subjects that underwent to gastric bypass surgery. Results are similar and showed reduction in consumption of morphine of 36 [74] and 40\% [75], respectively. Regarding opioid dependence, tDCS over the DLPFC could affect dopaminergic pathways, downregulating the reward system responses [58,76-79]. Alternately, tDCS stimulation of the DLPFC could strengthen executive functions, such as decision making and response inhibition $[29,80,81]$. Interestingly, these processes are reported also by studies interested in investigating the tDCS effects on food craving [82]. Indeed, the same neural system seems to be active for both natural rewards (e.g., food) and addictive drugs [69,83].

Regarding the localization of the stimulation, the role of M1 stimulation was shown by Khedr et al. [52]. In this study, an extracephalic montage with the anode over M1 resulted in similar findings to those achieved by Borckardt and collaborators in 2013 [53], counteracting the possibility that targeting both DLPFC and M1 could reduce the M1 stimulation effectiveness in reducing pain. However, Borckardt et al. found later in 2017 [55] that anodal tDCS over M1 caused an increased consumption of hydromorphone in contrast to what the same research group observed previously [53]. Beyond possible antagonistic effects of DLPFC downregulation action on M1 stimulation, authors did not exclude experimenter effects linked to the single-blind design they used in the 2013 trial [53]. This suggests the need for future studies of more rigorous methodology (e.g., double-blind design) to improve results' reliability. To this regard, Dubois et al. [51], considering both PCA morphine consumption and pain scores, did not observe statistically different effects between the three stimulation conditions (i.e., anodal, cathodal or sham) when applied over the left DLPFC. The relatively underpowered number of patients for each stimulation group (20 patients for anodal stimulation; 20 patients for cathodal stimulation; 19 patients for sham stimulation) could at least in part 
underlie the findings, leading to a type II error. Moreover, this trial is the only one among those retrieved that used a current intensity of $1 \mathrm{~mA}$, which may explain, in part, the lack of significant results. Prospectively, standardizing stimulation parameters among studies could help to compare different trials effectively.

A final important consideration here is the large variation of the location of the other return electrode. For instance, for M1 stimulation, there were four different montages (considering the return electrode: supraorbital [SO], somatosensory, DLPFC and extracephalic). It should be noted that the return electrode is critical for the induced current and thus the potential clinical effects. In a previous pain-modeling study, we showed that changing the return electrode resulted in no significant results and current was significantly displaced [84].

\section{tDCS to prevent \& treat postoperative pain}

Surgical procedures

To assess the possible analgesic effects of tDCS in the postoperative population, we included all studies conducted in a surgical setting without filtering the results based on surgical technique and procedures. This resulted in a variety of interventions (i.e., endoscopic retrograde cholangiopancreatography [54], total knee arthroplasty [52,53,55], lumbar spine surgeries - fusion procedures, decompression, herniated disc repair, laminectomy and hardware revision $[51,56]$ - and hallux valgus surgery [57]). It is possible to infer that the surgical interventions were not standardized for all the subjects, which might be considered a confounding factor, since different surgical procedures involve distinct organs and tissues which create a variety of patterns of nociceptor sensitization and differences in the quality, location and intensity of postoperative pain. Hence, standardizing surgical procedures in these kinds of studies seems to be a crucial factor since it improves the reliability of the experiments and helps the interpretation of the observed tDCS effects. However, it may be an important limitation for subjects' inclusion and poorly realistic with surgical practice.

\section{Components of pain}

The onset of pain is an important factor to consider when tDCS is performed. Even though acute and chronic pain involves common pathways, long-term experience of pain may induce changes in brain processes that would not be provoked by acute pain [85]. Acute pain involves mainly the somatosensory cortex during injury and inflammatory processes as a transient adaptive response [86], while chronic pain induces plastic changes in both the limbic system and cortical structures such as the somatosensory and prefrontal cortex [87]. Studies performed in populations that experience chronic pain show cortical reorganization as a possible factor to pain perpetuation [86,87]. Therefore, pain chronicity could affect the tDCS modulatory effects. The majority of studies (five out of seven) found in this review did not describe the exact onset of pain, even though it is reasonable to assume that most of the patients were facing chronic pain, which constitutes an indication to an elective surgical procedure. The effects of $\mathrm{tDCS}$ on chronic pain have already been extensively described in the literature in various pathologies [88,89]; however, future studies should control for pain chronicity to better clarify the tDCS analgesic effects.

Moreover, not only the onset of pain but also the emotional components of pain seem to be crucial. Among the studies retrieved by this review, only the one of Ribeiro et al. [57] assessed the pain-related catastrophic thinking using the Brazilian Portuguese Catastrophizing Scale, showing a medium effect size of 0.37 following the intervention. This suggests the need for future studies to control for the catastrophic behavior, which represents a stable and independent from physical impairment [90] feature of several pain syndromes such as rheumatoid arthritis, fibromyalgia and low back pain [91-95]. Particularly, the catastrophizing thinking, being the tendency to magnify the threat value of pain, to feel helpless and to be unable to prevent or inhibit pain-related thoughts, could negatively affect how subjects perceive pain and increase pain-related disability, emotional distress as well as avoidance behavior [96-99]. Previous evidence in patients with fibromyalgia showed a relationship between catastrophizing and the activity of brain areas involved in the anticipation of pain, attention to pain and painrelated emotional processing, mainly the medial frontal cortex, the DLPFC, the anterior cingulated cortex and amygdala [100]. Since these areas underlie top-down cognitive and emotional modulation of pain [101], abnormalities in descending modulatory systems in patients with chronic pain could explain these results $[102,103]$ and those related to hyperexcitability of pain pathways [85]. Therefore, tDCS could effectively be applied to improve the disinhibited state of cortical neural circuits, resulting in a better control of both pain [31,57] and pain-related emotions [104]. 


\section{Follow-up time}

The short follow-up time adopted by the seven papers does not allow us to infer about perpetuation effects of stimulation and triggers the possibility of higher demand of analgesics at discharge. Also, none of the studies discussed if the statistical differences observed in pain scales after tDCS could be translated to a clinical meaningful difference.

\section{tDCS to treat opioid dependence}

Wang et al. [58] provided promising results, showing that active tDCS significantly reduced craving scores when heroin-addicted subjects were exposed to videos of both heroin injection and inhalation. However, some limits in this study must be discussed. First, subjects' heroin use history. Although authors controlled for the abstinence period, they did not consider the individual differences regarding heroin use history length. Indeed, we can speculate that few years of heroin addiction correspond to low level of chronicity, representing a protective factor toward structural and functional alterations of brain areas involved in drug addiction [105-107]. This, in turn, could influence the tDCS effects. Indeed, a growing literature is reporting a certain individual variability of tDCS effects, suggesting the need of tailoring personalized stimulation protocols [108-111]. Second, another possible confounder factor is related to difference in pretreatment craving scores between the two experimental groups. The fact that the tDCS group had a higher pretreatment craving score than the sham group, even though the difference was not statistically significant, could underlie a greater sensitivity of subjects in tDCS group to stimulation's effects and explain in part the results. Although the study is a Phase II trial and therefore does not aim at external validity, the extremely homogeneous characteristics of the sample (e.g., only male subjects, same period of abstinence) do not allow for the generalizability of the results in other populations. Future studies need to test tDCS effects on opioid consumption considering larger samples and both genders. Moreover, even though presenting real video or images of craving-induced stimuli to explore tDCS effects on craving is common [82,112,113], it is important to consider whether this could trigger an after-study increase in exposure to drugs. Exposing subjects with an addiction history to addiction-related stimuli could result in an increased risk of relapse [69]. Hence, alternative stimuli should be taken into account to ensure safer experimental settings.

\section{Limitations}

The main limitation in this review is the number of articles and the important heterogeneity among them precluding drawing more firm conclusions regarding the use of tDCS to prevent and treat opioid dependence in postsurgical opioid dependence. However, this is an important emerging research area that can have a significant impact in public health, given the opioid epidemic and given the lack of cost-effective alternatives. In addition, most of the studies using different strategies show significant effects; thus it is important to discuss these initial studies as to help researchers designing future trials in this topic.

\section{Conclusion \& future perspective}

Despite the limited number of studies assessing tDCS effects in controlling postsurgical pain and opioid dependence, the results of this review showed encouraging directions. However, some considerations should be taken into account for future clinical trials testing the effects of tDCS in the field of surgical pain and opioid dependence. First, clinical research focused on pain management is difficult to carry out: high drop-out rates and several ethical concerns are typical of these kinds of studies. The growing amount of studies using home-based protocols [114-117] may overcome this limitation; especially given the importance to increase the number of sessions to enhance their clinical effects [84]. Furthermore, individuals suffering from pain are extremely vulnerable to placebo effect [118,119]. Thus, a run-in-phase approach and more rigorous methodologies, including double-blind designs, should be considered. Longer follow-ups are also needed to evaluate the long-term effects of stimulation in postsurgical pain and opioid dependence. Finally, it is important to find out which strategy would be most effective, but also certainly preventive strategies are worthwhile to be considered with priority. We recently showed that tDCS can indeed prevent stress-induced pain in animals [120]. Based on this review, even if the available data are still limited, tDCS seems to represent a safe and inexpensive tool to prevent and treat postsurgical pain and analgesic overuse. 


\section{Executive summary}

- Nowadays, opioid dependence represents a major problem, with high mortality rates and costs for health systems.

- Opioid therapies are mainly prescribed to treat postoperative pain.

- The burdens of traditional pharmacological interventions to deal with opioid dependence have led to the search of alternative treatments.

- Transcranial direct current stimulation, being safe, simple, inexpensive and a well-tolerated neuromodulation technique, has demonstrated positive effects on both chronic pain and opioid addiction.

- The eight studies retrieved by this review showed that M1 and the dorsolateral prefrontal cortex might be preferential stimulation targets.

- Despite the limitations, our review provides promising data regarding the role of transcranial direct current stimulation in enhancing top-down regulation processes that interfere with pain-related areas and the reward system.

\section{Acknowledgements}

The authors are thankful to H Rafferty (Spaulding Neuromodulation Laboratory, Harvard Medical School, Boston, MA, USA) for her suggestions throughout the writing of this review and to C Mita (Countway Library, Harvard Medical School, Boston, MA, USA) for the development of search descriptors.

Financial \& competing interests disclosure

The authors have no relevant affiliations or financial involvement with any organization or entity with a financial interest in or financial conflict with the subject matter or materials discussed in the manuscript. This includes employment, consultancies, honoraria, stock ownership or options, expert testimony, grants or patents received or pending, or royalties.

No writing assistance was utilized in the production of this manuscript.

\section{References}

Papers of special note have been highlighted as: • of interest

1. Rudd RA, Seth P, David F, Scholl L. Increases in drug and opioid-involved overdose deaths - United States, 2010-2015. MMWR Morb. Mortal. Wkly. Rep. 65(5051), 1445-1452 (2016).

2. Elzey MJ, Barden SM, Edwards ES. Patient characteristics and outcomes in unintentional, non-fatal prescription opioid overdoses: a systematic review. Pain Physician 19(4), 215-228 (2016).

3. Humphreys K. Avoiding globalisation of the prescription opioid epidemic. Lancet 390(10093), 437-439 (2017).

4. Brat GA, Agniel D, Beam A et al. Postsurgical prescriptions for opioid naive patients and association with overdose and misuse: retrospective cohort study. BMJ 360 (2018).

5. Valenstein M, Bair MJ, Ganoczy D, Mccarthy JF, Ilgen MA, Blow FC. Association between opioid prescribing patterns and opioid overdose-related deaths. JAMA 305(13), 1315-1321 (2011).

6. Veilleux JC, Colvin PJ, Anderson J, York C, Heinz AJ. A review of opioid dependence treatment: pharmacological and psychosocial interventions to treat opioid addiction. Clin. Psychol. Rev. 30(2), 155-166 (2010).

7. Judd LL, Marston MG, Attkisson C et al. Effective medical treatment of opiate addiction. J. Am. Med. Assoc. 280(22), 1936-1943 (1998).

8. Sees KL, Delucchi KL, Masson C et al. Methadone maintenance vs 180-day psychosocially enriched detoxification for treatment of opioid dependence: a randomized controlled trial. JAMA 283(10), 1303-1310 (2000).

9. Zaric GS, Barnett PG, Brandeau ML. HIV transmission and the cost-effectiveness of methadone maintenance. Am. J. Public Health 90(7), 1100-1111 (2000).

10. Ward J, Hall W, Mattick RP. Role of maintenance treatment in opioid dependence. Lancet 353(9148), 221-226 (1999).

11. Weinrich M, Stuart M. Provision of methadone treatment in primary care medical practices: review of the scottish experience and implications for us policy. JAMA J. Am. Med. Assoc. 283(10), 1343-1348 (2000).

12. Caplehorn JRM, Drummer OH. Mortality associated with new south wales methadone programs in 1994: lives lost and saved. Med. J. Aust. 170(3), 104-109 (1999).

13. Perret G, Deglon JJ, Kreek MJ, Ho A, La Harpe R. Lethal methadone intoxications in geneva, switzerland, from 1994 to 1998. Addiction 95(11), 1647-1653 (2000).

14. Pani PP, Maremmani I, Pirastu R, Tagliamonte A, Gessa GL. Buprenorphine: a controlled clinical trial in the treatment of opioid dependence. Drug Alcohol Depend. 60(1), 39-50 (2000). 
15. Finney JW, Moos RH, Timko C. The course of treated and untreated substance use disorders: remission and resolution, relapse and mortality. In: BS McCrady, EE Epstein (Eds). Addictions: A Comprehensive Guidebook (2nd Edition). Oxford University Press, USA, 108-131 (2013).

16. Lupi M, Martinotti G, Santacroce R et al. Transcranial direct current stimulation in substance use disorders: a systematic review of scientific literature. J. ECT 33(3), 203-209 (2017).

17. Terraneo A, Leggio L, Saladini M, Ermani M, Bonci A, Gallimberti L. Transcranial magnetic stimulation of dorsolateral prefrontal cortex reduces cocaine use: a pilot study. Eur. Neuropsychopharmacol. 26(1), 37-44 (2016).

18. Shahbabaie A, Ebrahimpoor M, Hariri A et al. Transcranial dc stimulation modifies functional connectivity of large-scale brain networks in abstinent methamphetamine users. Brain Behav. 8(3), e00922 (2018).

19. Fregni F, Liguori P, Fecteau S, Nitsche MA, Pascual-Leone A, Boggio PS. Cortical stimulation of the prefrontal cortex with transcranial direct current stimulation reduces cue-provoked smoking craving: a randomized, sham-controlled study. J. Clin. Psychiatry 69(1), 32-40 (2008).

20. Vitor de Souza Brangioni MC, Pereira DA, Thibaut A, Fregni F, Brasil-Neto JP, Boechat-Barros R. Effects of prefrontal transcranial direct current stimulation and motivation to quit in tobacco smokers: a randomized, sham controlled, double-blind trial. Front. Pharmacol. 9, 14 (2018).

21. Nitsche MA, Paulus W. Excitability changes induced in the human motor cortex by weak transcranial direct current stimulation. $J$. Physiol. 527 Pt 3, 633-639 (2000).

22. Priori A, Berardelli A, Rona S, Accornero N, Manfredi M. Polarization of the human motor cortex through the scalp. Neuroreport 9(10), 2257-2260 (1998).

23. Creutzfeldt OD, Fromm GH, Kapp H. Influence of transcortical d-c currents on cortical neuronal activity. Exp. Neurol. 5(6), 436-452 (1962).

24. Purpura DP, McMurtry JG. Intracellular activities and evoked potential changes during of motor cortex. Neurophysiol. 28(1), 166-185 (1965).

25. Gartside IB. Mechanisms of sustained increases of firing rate of neurones in the rat cerebral cortex after polarization: role of protein synthesis. Nature 220(5165), 383-384 (1968).

26. Conti CL, Nakamura-Palacios EM. Bilateral transcranial direct current stimulation over dorsolateral prefrontal cortex changes the drug-cued reactivity in the anterior cingulate cortex of crack-cocaine addicts. Brain Stimul. 7(1), 130-132 (2014).

27. Boggio PS, Liguori P, Sultani N, Rezende L, Fecteau S, Fregni F. Cumulative priming effects of cortical stimulation on smoking cue-induced craving. Neurosci. Lett. 463(1), 82-86 (2009).

28. Xu J, Fregni F, Brody AL, Rahman AS. Transcranial direct current stimulation reduces negative affect but not cigarette craving in overnight abstinent smokers. Front. Psychiatry 4, 112 (2013).

29. Fecteau S, Agosta S, Hone-Blanchet A et al. Modulation of smoking and decision-making behaviors with transcranial direct current stimulation in tobacco smokers: a preliminary study. Drug Alcohol Depend. 140, 78-84 (2014).

30. Boggio PS, Sultani N, Fecteau S et al. Prefrontal cortex modulation using transcranial dc stimulation reduces alcohol craving: a double-blind, sham-controlled study. Drug Alcohol Depend. 92(1-3), 55-60 (2008).

31. Valle A, Roizenblatt $S$, Botte $S$ et al. Efficacy of anodal transcranial direct current stimulation (tDCS) for the treatment of fibromyalgia: results of a randomized, shamcontrolled longitudinal clinical trial. J. Pain Manag. 2(3), 353-361 (2009).

32. García-Larrea L, Peyron R, Mertens P et al. Electrical stimulation of motor cortex for pain control: a combined pet-scan and electrophysiological study. Pain 83(2), 259-273 (1999).

33. Bolognini N, Olgiati E, Maravita A, Ferraro F, Fregni F. Motor and parietal cortex stimulation for phantom limb pain and sensations. Pain 154(8), 1274-1280 (2013).

34. Fregni F, Boggio PS, Lima MC et al. A sham-controlled, Phase II trial of transcranial direct current stimulation for the treatment of central pain in traumatic spinal cord injury. Pain 122(1-2), 197-209 (2006).

35. Mendonca ME, Santana MB, Baptista AF et al. Transcranial DC stimulation in fibromyalgia: optimized cortical target supported by high-resolution computational models. J. Pain 12(5), 610-617 (2011).

36. Soler MD, Kumru H, Pelayo R et al. Effectiveness of transcranial direct current stimulation and visual illusion on neuropathic pain in spinal cord injury. Brain 133(9), 2565-2577 (2010).

37. Mendonca ME, Simis M, Grecco LC, Battistella LR, Baptista AF, Fregni F. Transcranial direct current stimulation combined with aerobic exercise to optimize analgesic responses in fibromyalgia: a randomized placebo-controlled clinical trial. Front. Hum. Neurosci. 68, 10 (2016).

38. Cummiford CM, Nascimento TD, Foerster BR et al. Changes in resting state functional connectivity after repetitive transcranial direct current stimulation applied to motor cortex in fibromyalgia patients. Arthritis Res. Ther. 18(1), 40 (2016).

39. Polanía R, Paulus W, Nitsche M. Modulating cortico-striatal and thalamo-cortical functional connectivity with transcranial direct current stimulation. Hum. Brain Mapp. 33(10), 2499-2508 (2012). 
40. Chiou R, Lee H, Chang C, Lin K, CC K. Epidural motor cortex stimulation suppresses somatosensory evoked potentials in the primary somatosensory cortex of the rat. Brain Res. 42-50 (2012).

41. Knotkova H, Nitsche MA, Cruciani RA. Putative physiological mechanisms underlying tDCS analgesic effects. Front. Hum. Neurosci. 7(September), 1-5 (2013).

42. DaSilva A, Granziera C, Hadjikhani J. Thickening in the somatosensory cortex of patients with migraine. Neurology 69(21), 1990-1995 (2007).

43. Warner R, Hudson-Howard L, Johnston C, Skolnick M. Serotonin involvement in analgesia induced by transcranial electrostimulation. Life Sci. 46(16), 1131-1138 (1990).

44. Malin D, Lake J, Hamilton R, Skolnick M. Augmented analgesic effects of l-tryptophan combined with low current transcranial electrostimulation. Life Sci. 47(4), 263-267 (1990).

45. Skolnick M, Wilson O, Hamilton R et al. Low current electrostimulation produces naloxone-reversible analgesia in rats. Stereotact. Funct. Neurosurg. 53(2), 125-140 (1989).

46. DosSantos MF, Martikainen IK, Nascimento TD et al. Building up analgesia in humans via the endogenous $\mu$-opioid system by combining placebo and active tdcs: a preliminary report. PLoS ONE 9(7), 1-9 (2014).

47. Moher D, Shamseer L, Clarke M et al. Preferred reporting items for systematic review and meta-analysis protocols (prisma-p) 2015 statement. Syst. Rev. 4(1), 1 (2015).

48. Shamseer L, Moher D, Clarke M et al. Preferred reporting items for systematic review and meta-analysis protocols (prisma-p) 2015: elaboration and explanation. BMJ. 350, g7647 (2015).

49. NIH's definition of a clinical trial | grants.nih.gov

50. Ouzzani M, Hammady H, Fedorowicz Z, Elmagarmid A. Rayyan - a web and mobile app for systematic reviews. Syst. Rev. 5(1), 210 (2016).

51. Dubois PE, Ossemann M, de Fays $\mathrm{K}$ et al. Postoperative analgesic effect of transcranial direct current stimulation in lumbar spine surgery: a randomized control trial. Clin. J. Pain 29(8), 696-701 (2013).

- To our knowledge, this is the first study investigating the effects of transcranial direct current stimulation (tDCS) in postoperative pain reduction in subjects who received lumbar spine surgery.

52. Khedr EM, Sharkawy ESA, Attia AMA, Ibrahim Osman NM, Sayed ZM. Role of transcranial direct current stimulation on reduction of postsurgical opioid consumption and pain in total knee arthroplasty: double randomized clinical trial. Eur. J. Pain 21(8), 1355-1365 (2017).

- Investigates the decrease in pain by using an extraencephalic montage, allowed us to discuss the parameters of stimulation.

53. Borckardt JJ, Reeves ST, Robinson SM et al. Transcranial direct current stimulation (tDCS) reduces postsurgical opioid consumption in total knee arthroplasty (TKA). Clin. J. Pain 29(11), 925-928 (2013).

- Explores the role of stimulation of motor cortex in reducing pain in subjects who underwent total knee arteroplasty.

54. Borckardt JJ, Romagnuolo J, Reeves ST et al. Feasibility, safety, and effectiveness of transcranial direct current stimulation for decreasing post-ERCP pain: a randomized, sham-controlled, pilot study. Gastrointest. Endosc. 73(6), 1158-1164 (2011).

- The methodology and objectives described in this study fit with the goals of this review.

55. Borckardt JJ, Reeves ST, Milliken C et al. Prefrontal versus motor cortex transcranial direct current stimulation (tDCS) effects on post-surgical opioid use. Brain Stimul. 10(6), 1096-1101 (2017).

- Investigates the potential therapeutic role of three different tDCS montages to treat postoperative pain.

56. Glaser J, Reeves ST, Stoll WD et al. Motor/prefrontal transcranial direct current stimulation (tDCS) following lumbar surgery reduces postoperative analgesia use. Spine (Phila. Pa. 1976) 41(10), 835-839 (2016).

- Explores the role of motor cortex stimulation in reducing pain after lumbar spine surgery. The authors also apply a higher current intensity than the one adopted in a previous study that investigated the same parameters in the same population.

57. Ribeiro H, Sesterhenn RB, De Souza A et al. Preoperative transcranial direct current stimulation: exploration of a novel strategy to enhance neuroplasticity before surgery to control postoperative pain. A randomized sham-controlled study. PLoS ONEdoi:10.1371/journal.pone.0187013 (2017) (Epub ahead of print).

- The only study retrieved that administrates a catastrophizing scale.

58. Wang Y, Shen Y, Cao X et al. Transcranial direct current stimulation of the frontal-parietal-temporal area attenuates cue-induced craving for heroin. J. Psychiatr. Res. 79, 1-3 (2016).

- The only one retrieved by the search ' $t D C S$ to treat opioid dependence'. The lack of studies covering this issue may stimulate the development of research protocols focusing on craving reduction.

59. Bikson M, Grossman P, Thomas C et al. Safety of transcranial direct current stimulation: evidence based update 2016. Brain Stimul. 9(5), 641-661 (2016).

60. Aron AR, Robbins TW, Poldrack RA. Inhibition and the right inferior frontal cortex. Trends Cogn. Sci. 8(4), 170-177 (2004). 
61. Berkman ET, Burklund L, Lieberman MD. Inhibitory spillover: intentional motor inhibition produces incidental limbic inhibition via right inferior frontal cortex. Neuroimage 47(2), 705-712 (2009).

62. Boggio PS, Zaghi S, Fregni F. Modulation of emotions associated with images of human pain using anodal transcranial direct current stimulation (tDCS). Neuropsychologia 47(1), 212-217 (2009).

63. Boggio PS, Zaghi S, Lopes M, Fregni F. Modulatory effects of anodal transcranial direct current stimulation on perception and pain thresholds in healthy volunteers. Eur. J. Neurol. 15(10), 1124-1130 (2008).

64. Fierro B, De Tommaso M, Giglia F, Giglia G, Palermo A, Brighina F. Repetitive transcranial magnetic stimulation (RTMS) of the dorsolateral prefrontal cortex (DLPFC) during capsaicin-induced pain: modulatory effects on motor cortex excitability. Exp. Brain Res. 203(1), 31-38 (2010).

65. Brighina F, Piazza A, Vitello G et al. RTMS of the prefrontal cortex in the treatment of chronic migraine: a pilot study. J. Neurol. Sci. 227(1), 67-71 (2004).

66. McBride D, Barrett SP, Kelly JT, Aw A, Dagher A. Effects of expectancy and abstinence on the neural response to smoking cues in cigarette smokers: an FMRI study. Neuropsychopharmacology 31(12), 2728-2738 (2006).

67. Di Chiara G, Bassareo V, Fenu S et al. Dopamine and drug addiction: the nucleus accumbens shell connection. Neuropharmacology 47, 227-241 (2004).

68. Nestler EJ. Is there a common molecular pathway for addiction? Nat. Neurosci. 8(11), 1445-1449 (2005).

69. Tang DW, Fellows LK, Small DM, Dagher A. Food and drug cues activate similar brain regions: a meta-analysis of functional mri studies. Physiol. Behav. 106(3), 317-324 (2012).

70. Mottaghy FM, Krause BJ, Kemna LJ et al. Modulation of the neuronal circuitry subserving working memory in healthy human subjects by repetitive transcranial magnetic stimulation. Neurosci. Lett. 280(3), 167-170 (2000).

71. Seminowicz DA, Davis KD. Cortical responses to pain in healthy individuals depends on pain catastrophizing. Pain 120(3), 297-306 (2006).

72. Knotkova H, Cruciani RA. Non-invasive transcranial direct current stimulation for the study and treatment of neuropathic pain. Trends Mol. Biol. 617, 505-515 (2010).

73. Lang N, Siebner HR, Ward NS et al. How does transcranial DC stimulation of the primary motor cortex alter regional neuronal activity in the human brain? Eur. J. Neurosci. 22(2), 495-504 (2005).

74. Borckardt JJ, Reeves ST, Weinstein M et al. Significant analgesic effects of one session of postoperative left prefrontal cortex repetitive transcranial magnetic stimulation: a replication study. Brain Stimul. 1(2), 122-127 (2008).

75. Borckardt JJ, Weinstein M, Reeves ST et al. Postoperative left prefrontal repetitive transcranial magnetic stimulation reduces patient-controlled analgesia use. Anesthesiology 105(3), 557-562 (2006).

76. Nitsche MA, Lampe C, Antal A et al. Dopaminergic modulation of long-lasting direct current-induced cortical excitability changes in the human motor cortex. Eur. J. Neurosci. 23(6), 1651-1657 (2006).

77. Pascoli V, Terrier J, Espallergues J, Valjent E, O’Connor EC, Lüscher C. Contrasting forms of cocaine-evoked plasticity control components of relapse. Nature 509(7501), 459-464 (2014).

78. Pogarell O, Koch W, Pöpperl G et al. Striatal dopamine release after prefrontal repetitive transcranial magnetic stimulation in major depression: preliminary results of a dynamic [123i] IBZM SPECT study. J. Psychiatr. Res. 40(4), 307-314 (2006).

79. Strafella AP, Paus T, Barrett J, Dagher A. Repetitive transcranial magnetic stimulation of the human prefrontal cortex induces dopamine release in the caudate nucleus. J. Neurosci. 21(RC157), 1-4 (2001).

80. Ouellet J, McGirr A, Van den Eynde F, Jollant F, Lepage M, Berlim MT. Enhancing decision-making and cognitive impulse control with transcranial direct current stimulation (tDCS) applied over the orbitofrontal cortex (OFC): a randomized and sham-controlled exploratory study. J. Psychiatr. Res. 69, 27-34 (2015).

81. Soltaninejad Z, Nejati V, Ekhtiari H. Effect of anodal and cathodal transcranial direct current stimulation on DLPFC on modulation of inhibitory control in ADHD. J. Atten. Disord. pii: 108705471561879 (2015) (Epub ahead of print).

82. Fregni F, Orsati F, Pedrosa W et al. Transcranial direct current stimulation of the prefrontal cortex modulates the desire for specific foods. Appetite 51(1), 34-41 (2008).

83. Di Chiara G. Drug addiction as dopamine-dependent associative learning disorder. Eur. J. Pharmacol. 375(1-3), 13-30 (1999).

84. Mendonca ME, Santana MB, Baptista AF et al. Transcranial dc stimulation in fibromyalgia: optimized cortical target supported by high-resolution computational models. J. Pain 12(5), 610-617 (2011).

85. Caumo W, Deitos A, Carvalho $\mathrm{S}$ et al. Motor cortex excitability and BDNF levels in chronic musculoskeletal pain according to structural pathology. Front. Hum. Neurosci. 10, 357 (2016).

86. Antal A, Brepohl N, Poreisz C, Boros K, Csifcsak G, Paulus W. Transcranial direct current stimulation over somatosensory cortex decreases experimentallyinduced acute pain perception. Clin. J. Pain 24(1), 56-63 (2008).

87. Flor H. Cortical reorganisation and chronic pain: implications for rehabilitation. J. Rehabil. Med. 35(suppl. 41), 66-72 (2003). 
88. Fregni F, Freedman S, Pascual-Leone A. Recent advances in the treatment of chronic pain with non-invasive brain stimulation techniques. Lancet Neurol. 6(2), 188-191 (2007).

89. Lima MC, Fregni F. Motor cortex stimulation for chronic pain: systematic review and meta-analysis of the literature. Neurology 70(24), 2329-2337 (2008).

90. Severeijns R, Vlaeyen WS, Van Den Hout A, Weber EJ. Pain catastrophizing predicts pain intensity, disability, and psychological distress independent of the level of physical impairment. Clin. J. Pain 17(2), 165-172 (2001).

91. Edwards RR, Cahalan C, Mensing G, Smith M, Haythornthwaite JA. Pain, catastrophizing and depression in the rheumatic diseases. Nat. Rev. Rheumatol. 7(4), 216-224 (2011).

92. Keefe FJ, Brown GK, Wallston KA, Caldwell DS. Coping with rheumatoid arthritis pain: catastrophizing as a maladaptive strategy. Pain 37(1), 51-56 (1989).

93. Flor H, Behle DJ, Birbaumer N. Assessment of pain-related cognitions in chronic pain patients. Behav. Res. Ther. 31(1), 63-73 (1993).

94. Martin MY, Bradley LA, Alexander RW et al. Coping strategies predict disability in patients with primary fibromyalgia. Pain 68(1), 45-53 (1996).

95. Sullivan MJ, Thorn B, Haythornthwaite JA et al. Theoretical perspectives on the relation between catastrophizing and pain. Clin. J. Pain 17(1), 52-64 (2001).

96. McCracken LM. 'Attention' to pain in persons with chronic pain: a behavioral approach. Behav. Ther. 28(2), 271-284 (1997).

97. Asmundson GJ, Kuperos JL, Ron Norton G. Do patients with chronic pain selectively attend to pain-related information?: preliminary evidence for the mediating role of fear. Pain 72(1-2), 27-32 (1997).

98. Van Damme S, Crombez G, Eccleston C. Disengagement from pain: the role of catastrophic thinking about pain. Pain 107(1-2), 70-76 (2004).

99. Quartana PJ, Campbell CM, Edwards RR. Pain catastrophizing: a critical review. Expert Rev. Neurother. 9(5), 745-758 (2009).

100. Gracely RH, Geisser ME, Giesecke T et al. Pain catastrophizing and neural responses to pain among persons with fibromyalgia. Brain 127(4), 835-843 (2004).

101. Bushnell MC, Čeko M, Low LA. Cognitive and emotional control of pain and its disruption in chronic pain. Nat. Rev. Neurosci. 14(7), 502-511 (2013).

102. Ossipov MH, Dussor GO, Porreca F. Central modulation of pain. J. Clin. Invest. 120(11), 3779-3787 (2010).

103. Porreca F, Ossipov MH, Gebhart G. Chronic pain and medullary descending facilitation. Trends Neurosci. 25(6), 319-325 (2002).

104. Lagueux E, Bourgault P, Tousignant-Laflamme Y. (475) effectiveness of transcranial direct current stimulation (tDCS) and graded motor imagery (GMI) on neuropathic pain of complex regional pain syndrome type I. J. Pain 16(4), S94 (2015).

105. Volkow ND, Fowler JS, Wang G-J. The addicted human brain viewed in the light of imaging studies: brain circuits and treatment strategies. Neuropharmacology 47, 3-13 (2004).

106. Wollman SC, Alhassoon OM, Hall MG et al. Gray matter abnormalities in opioid-dependent patients: a neuroimaging meta-analysis. Am. J. Drug Alcohol Abuse 43(5), 505-517 (2017).

107. Lin H-C, Wang P-W, Wu H-C, Ko C-H, Yang Y-H, Yen C-F. Altered gray matter volume and disrupted functional connectivity of dorsolateral prefrontal cortex in men with heroin dependence. Psychiatry Clin. Neurosci. 72(6), 435-444 (2018).

108. Lefaucheur J-P, Antal A, Ayache SS et al. Evidence-based guidelines on the therapeutic use of transcranial direct current stimulation (tDCS). Clin. Neurophysiol. 128(1), 56-92 (2017).

109. López-Alonso V, Fernández-Del-Olmo M, Costantini A, Gonzalez-Henriquez JJ, Cheeran B. Intra-individual variability in the response to anodal transcranial direct current stimulation. Clin. Neurophysiol. 126(12), 2342-2347 (2015).

110. Wiethoff S, Hamada M, Rothwell JC. Variability in response to transcranial direct current stimulation of the motor cortex. Brain Stimul. 7(3), 468-475 (2014).

111. Strube W, Bunse T, Malchow B, Hasan A. Efficacy and interindividual variability in motor-cortex plasticity following anodal tDCS and paired-associative stimulation. Neural. Plast. 2015, 1-10 (2015).

112. Goldman RL, Borckardt JJ, Frohman HA et al. Prefrontal cortex transcranial direct current stimulation (tDCS) temporarily reduces food cravings and increases the self-reported ability to resist food in adults with frequent food craving. Appetite 56(3), 741-746 (2011).

113. Uher R, Yoganathan D, Mogg A et al. Effect of left prefrontal repetitive transcranial magnetic stimulation on food craving. Biol. Psychiatry 58(10), 840-842 (2005).

114. Martens G, Lejeune N, O'Brien AT et al. Randomized controlled trial of home-based 4-week tDCS in chronic minimally conscious state. Brain Stimul. 11(5), 982-990 (2018).

115. Charvet LE, Kasschau M, Datta A et al. Remotely-supervised transcranial direct current stimulation (tDCS) for clinical trials: guidelines for technology and protocols. Front. Syst. Neurosci.9(March), 1-13 (2015).

116. Charvet L, Shaw M, Dobbs B et al. Remotely supervised transcranial direct current stimulation increases the benefit of at-home cognitive training in multiple sclerosis. Neuromodulation 383-389 2017 (2017). 
117. Kasschau M, Reisner J, Sherman K, Bikson M, Datta A, Charvet LE. Transcranial direct current stimulation is feasible for remotely supervised home delivery in multiple sclerosis. Neuromodulation 19(8), 824-831 (2016).

118. Bingel U, Wanigasekera $\mathrm{V}$, Wiech $\mathrm{K}$ et al. The effect of treatment expectation on drug efficacy: imaging the analgesic benefit of the opioid remifentanil. Sci. Transl. Med. 3(70), 70ra14 (2011).

119. Dobrila-Dintinjana R, Nacinović-Duletić A. Placebo in the treatment of pain. Coll. Antropol. 35(Suppl 2), 319-323 (2011).

120. Fregni F, Macedo IC, Spezia-Adachi LN et al. Transcranial direct current stimulation (tDCS) prevents chronic stress-induced hyperalgesia in rats. Brain Stimul. 11(2), 299-301 (2018). 